\title{
Handgelenk und Fingergelenke
}

\author{
Thomas Pillukat, Nikolas Stütz, Jörg van Schoonhoven, Herrmann Krimmer
}

\section{Zusammenfassung}

In ausgewählten Fällen bildet auch an Gelenken der Hand die endoprothetische Versorgung eine Alternative zur bisher bevorzugten Versteifung. Zu den wichtigen Vorraussetzungen zählen neben einer ausreichenden knöchernen Abstützung intakte KapselBand-Strukturen und gleitfähige Sehnen. Der Ersatz von Grund- und Mittelgelenken der Finger ist wegen ihrer erheblichen funktionellen Wertigkeit besonders erstrebenswert. Der Einsatz spezieller Materialien (z. B. Pyrocarbon) könnte die abriebbedingten Probleme(Implantatlockerung, Umgebungsreaktion) minimieren. Konsequente Nachbehandlungskonzepte tragen wesentlich zum maximalen Erfolg bei. Die Ulnakopfprothese wird nicht nur bei Veränderungen des distalen Radioulnargelenkes, sondern auch bei Instabilitäten eingesetzt. Nach fehlgeschlagenen resezierenden Operationsverfahren an der distalenUlna (Ulnakopf-Hemiresektion, Arthrodese des distalen Radioulnargelenkes nach Kapandji-Sauve) bietet sie eine Rückzugsmöglichkeit. Die bisher schlechten Langzeitresultate implantierter Handgelenksprothesen sprechen für eine strenge und zurückhaltende Indikationsstellung. Für umschriebene Veränderungen des Radiokarpal- und Mediokarpalgelenkes bieten sich alternativ Teilversteifungen an.

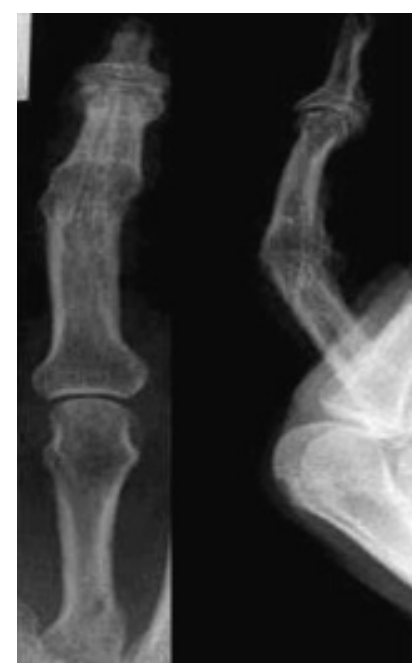

An Fingern und Handgelenk ist die Versteifung (Arthrodese) das bisher am häufigsten eingesetzte Verfahren, um nichtrekonstruierbare Gelenke zu versorgen

(Abb. 1).

In der Unfallchirurgie ist die Erhaltung oder Wiederherstellung verletzter Gelenke nicht immer möglich. Gerade an der Hand gestaltet sich die Rekonstruktion wegen der kleinen Dimensionen oft schwierig. Während sich Verluste oder Teilverluste von Gelenkflächen unmittelbar bemerkbar machen können, führen Fehlstellungen von Gelenkflächen und Bandinstabilitäten oft erst längerfristig zu Beschwerden. Typische Leitsymptome sind Schmerz, Instabilität, Deformität und Bewegungseinschränkung.

OP-JOURNAL 2003; 19: 210-215

(c) Georg Thieme Verlag Stuttgart · New York ziert und die Kraftentfaltung verbessert. Gleichzeitig geht die Beweglichkeit vollständig verloren, was funktionell und kosmetisch störend sein kann. Aus diesem Grund wird auch an den Fingern und am Handgelenk versucht, zerstörte Gelenke durch Endoprothesen zu ersetzen. Dabei sind die üblichen Grundsätze der Endoprothetik wie Infektfreiheit des OP-Gebietes, ausreichende Weichteildeckung und knöcherne Abstützung zu beachten. Besondere Aufmerksamkeit ist den Weichteilen und Funktionsgeweben der Hand zu widmen.

Die Kapsel-Bandstrukturen sollten intakt oder rekonstruierbar sein, um die Stabilität der Endoprothese zu gewährleisten. Die passive Beweglichkeit sollte nicht durch narbige Kontrakturen eingeschränkt sein. Beuge- und Strecksehnen müssen vorhanden sein und frei gleiten können, damit der durch den Gelenkersatz erzielte Gewinn an Beweglichkeit auch aktiv ausgenutzt werden kann.

Die bisher verfügbaren Implantate sind mechanisch deutlich weniger belastbar als die zu ersetzenden Gelenke. In Fällen hoher mechanischer Beanspruchung bei manuell tätigen Patienten ist daher kritisch abzuwägen, ob eine gut belastbare Arthrodese die bessere Alternative ist. Die Beachtung dieser Voraussetzungen schränkt die Gruppe von Patienten, die für eine endoprothetische Versorgung infrage kommen, erheblich ein.

\section{Prothesen der Fingergelenke}

\section{Grundlagen}

Abb.1 Arthrodese des Mittelgelenkes. Der ehemalige Gelenkspalt ist im Vergleich zum Grund- und Endgelenk knöchern überbrückt.

Bei diesem Vorgehen werden die veränderten Gelenkflächen entfernt und der ehemalige Gelenkspalt knöchern überbrückt. Dadurch wird der Schmerz redu-
Die funktionelle Wertigkeit der Fingergelenke ergibt sich aus ihrem Anteil am gesamten Bewegungsumfang. Dieser Anteil beträgt für
- das Endgelenk
- das Mittelgelenk $20 \%$
- das Grundgelenk 
Unter diesem Aspekt führt eine Versteifung des Endgelenkes nur zu einer geringen Einschränkung. Da Schmerzfreiheit und Stabilität durch eine Versteifung zuverlässig zu erzielen sind, wird eine endoprothetische Versorgung der Fingerendgelenke seltener durchgeführt.

Ein alloplastischer Ersatz von Grund- und Mittelgelenk ist dagegen erstrebenswert. Auch wenn der Anteil der Mittelgelenke am gesamten Bewegungsumfang mit $20 \%$ geringer ist als der der Grundgelenke (77\%), so zeigt sich doch eine deutliche Behinderung beim Umfassen von Gegenständen oder Greifen. Trotz dieser Funktionsverluste wird bei Fingergelenken die Arthrodese bevorzugt [5], weil die Resultate der endoprothetischen Versorgung, insbesondere der Mittelgelenke, bisher oft enttäuschend waren. Ursachen sind Abrieb mit Umgebungsreaktion und Prothesenlockerung. Der Weichteilmantel der Fingergelenke ist besonders schmächtig, in unmittelbarer Nachbarschaft finden sich komplexe bindegewebige Strukturen. Abriebinduzierte Umgebungsreaktionen führen nicht selten zu schwersten fibrotischen Veränderungen und Verklebung der Gleitschichten. Sie können den durch die Implantation erzielten Bewegungsumfang bis zur völligen Einsteifung einschränken.

\section{Indikation}

Hauptindikation für eine Fingergelenkprothese ist die posttraumatische Arthrose (Abb. 2) bei intaktem Weichteilmantel und funktionsfähigen Sehnen.

Seltener erfolgt die Implantation nach Gelenkflächenverlusten infolge einer Gelenkinfektion, sofern die Infektion sicher beseitigt ist.

\section{Implantate}

In der Vergangenheit sind unterschiedliche Prothesenformen (gekoppelt/ungekoppelt, zementiert/zementfrei) und Materialkombinationen eingesetzt worden. Die günstigsten Resultate scheinen ungekoppelte Systeme zu erzielen, die zementfrei implantiert werden und aus Materialien bestehen, die keinen wesentlichen Abrieb haben (Keramik, Pyrocarbon) [5]. Die Knochen im Fingerbereich verfügen im Vergleich $\mathrm{zu}$ anderen Körperregionen über eine nur geringe Substanz. Die zementfreie Implantation verursacht einen geringeren Substanzverlust. Er ist bei zementierten Prothesen

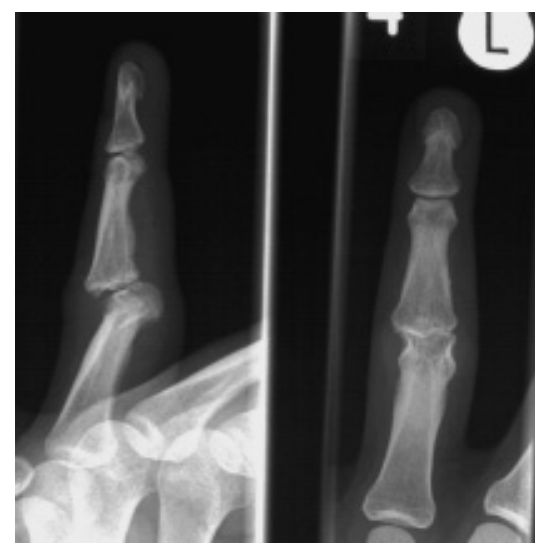

Abb. 2 Posttraumatische Arthrose eines Mittelgelenkes bei fixierter Subluxation. Das Gelenk ist in Streckstellung wackelsteif.

deutlich größer und erschwert die bei Implantatversagen erforderlichen Zweiteingriffe [3].

Eine besondere Bedeutung könnte in $\mathrm{Zu}$ kunft Pyrocarbonprothesen zukommen (Abb.3). Bei diesem Material handelt es sich um eine hochgradig abriebfeste Graphitlegierung, die ihre gute Biokompatibilität und Widerstandsfähigkeit bereits bei der Anwendung in künstlichen Herzklappen bewiesen hat [9].

\section{Voraussetzungen}

Bei den bevorzugten ungekoppelten Implantaten ist eine bindegewebige Stabili-

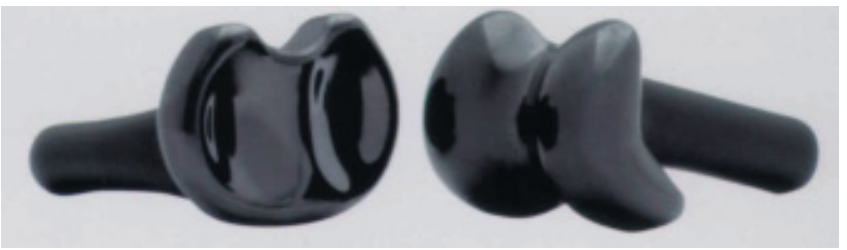

Abb. 3 Beispie einer Prothese des Mittelgelenkes mit Pyrocarbonbeschichtung (Fa. Ascension Orthopedics, Austin, Texas).

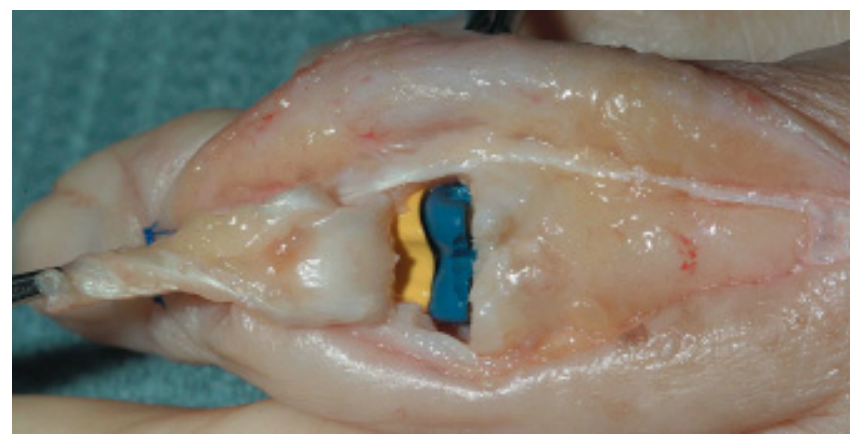

Abb.4 Probekomponenten einer Mittelgelenksprothese nach Reposition. Links im Bild der nach distal umgeschlagene Tractus intermedius mit Teilen der Streckaponeurose, der an der Mittelphalanx fixiert bleiben muss. Das gleiche Bild ergibt sich nach Implantation der endgültigen Prothese. 
a, b
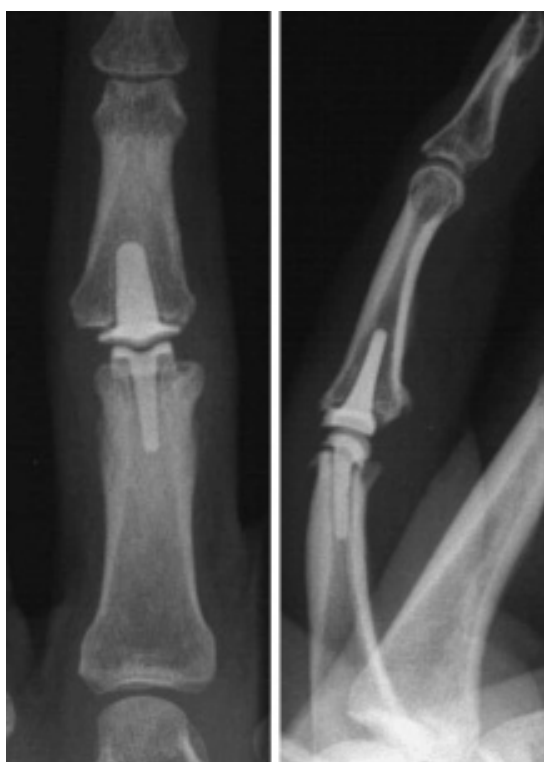

Abb. 5 a, b Korrekte Lage der Mittelgelenksprothese im Röntgenbild. Der Abstand zwischen Prothesenschaft und Grundphalanx entsteht durch die strahlentransparente Pyrocarbonbeschichtung und darf nicht als Lockerung oder Fehllage interpretiert werden.

Nach Aufweiten des Markraumes wird die proximale Probekomponente in passender Größe in den Knochen eingebracht. Anschließend wird die distale Gelenkfläche in gleicher Weise anhand von Sägeschablonen reseziert und die passende distale Probe-Prothesenkomponente ebenfalls provisorisch eingebracht. Dann erfolgt die Reposition des Gelenkes (Abb.4). Gegebenenfalls ist eine Nachresektion erforderlich. Anschließend werden die passenden endgültigen Prothesenkomponenten zementfrei formschlüssig (,press fit“) implantiert.

Nach Röntgenkontrolle (Abb.5au.b) wird die Streckaponeurose mit monofilem Faden fortlaufend genäht und die Haut verschlossen.

\section{Nachbehandlung}

In der postoperativen Phase richtet sich die Aufmerksamkeit darauf, Beweglichkeit und Gleitfähigkeit der Nachbarstrukturen (Bandapparat, Strecksehnen und Beugesehnen) zu erhalten. Nur so lässt sich der erzielte Bewegungsumfang sichern. $\mathrm{Zu}$ diesem Zweck sind differenzierte Nachbehandlungsregime entwickelt worden unter Einsatz angepasster ergotherapeutischer Schienen [9]. 8 Wochen nach der Implantation ist die Behandlung abgeschlossen. Angestrebt wird als Bewegungsumfang die vollstän-

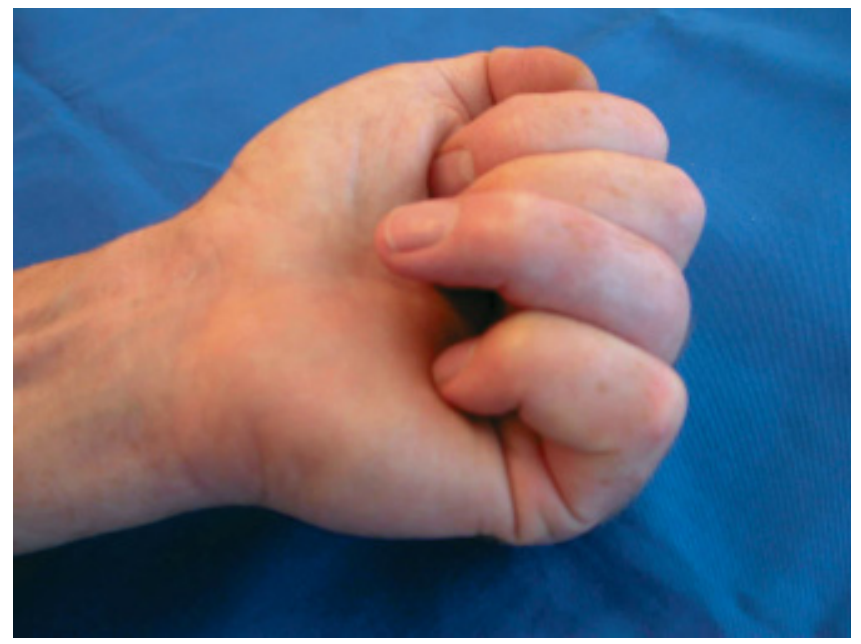

Abb. 6 Klinisches Bild nach Implantation einer Mittelgelenksprothese in den Ringfinger. Die Beugung im Mittelgelenk beträgt $70^{\circ}$.

dige Streckung und eine maximale Beugung von $70^{\circ}\left(0 / 0 / 70^{\circ}\right)$ (Abb. 6 )

\section{Ergebnisse}

Unsere Serie [9] implantierter Pyrocarbon-Prothesen für das Mittelgelenk ergab eine ausgeprägte Schmerzreduktion wie bei einer Arthrodese. Die Beweglichkeit der Mittelgelenke verbesserte sich von 0/28/51 und einem Bewegungsumfang (range of motion, ROM) von $21^{\circ}$ auf post-

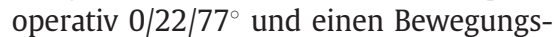
umfang (ROM) von 55. Die Mehrheit der Patienten ( $>80 \%$ ) war mit dem Resultat der Implantation zufrieden und sehr zufrieden. Alle Patienten waren ein Jahr nach der Operation schmerzfrei. In einem Nachbeobachtungszeitraum von durchschnittlich 360 Tagen traten keine Implantatfrakturen oder Schaftlockerungen auf.

\section{Prothesen des Ulnakopfes}

\section{Grundlagen}

Die Drehbewegung des Unterarmes um seine Längsachse erfordert das reibungslose Zusammenspiel zweier mechanisch gekoppelter Gelenke, des proximalen und des distalen Radioulnargelenkes. Der Umfang dieser Bewegung beträgt bis zu $175^{\circ}$. Veränderungen am distalen Radioulnargelenk (Abkürzung: DRUG) verursachen schmerzhafte Einschränkungen der Unterarmdrehung mit Kraftverlust in der Hand und erheblicher funktioneller Beeinträchtigung der gesamten oberen Extremität.

\section{Indikation}

Eine direkte Zerstörung des distalen Radioulnargelenkes kann durch intaartikuläre Frakturen des Ulnakopfes oder der
Incisura ulnaris des distalen Radius entstehen. Die posttraumatische Arthrose tritt als Folge von in Fehlstellung verheilten extraartikulären distalen Radiusfrakturen oder bei chronischer Instabilität des DRUG nach Verletzungen des ulnokarpalen Bandkomplexes (TFCC $=$ triangulärer fibrocartilaginärer Komplex $=\mathrm{Fa}-$ serknorpelplatte zwischen Ulnakopf und proximaler Handwurzelreihe) auf.

Als operative Verfahren stehen bisher die Ulnakopfresektion, die Hemiresektionsarthroplastik des Ulnakopfes oder die Arthrodese des DRUG mit Segmentresektion aus der distalen Ulna (Operation nach Kapandji-Sauve) zur Verfügung. Durch Unterbrechung der knöchernen Integrität der Ulna ist bei allen drei Verfahren die physiologische Abstützung der Ulna nicht mehr gewährleistet und gleichzeitig die Funktion der Weichteile beeinträchtigt, die das distale Radioulnargelenk stabilisieren [8]. Dies kann zu schmerzhaften Instabilitäten des distalen Ulnaendes führen. Die Ulnakopfprothese soll die Abstützung und die Rotation des Radius im DRUG wiederherstellen und den stabilisierendenden Weichteilen (TFCC, Membrana interossea, M pronator quadratus) die notwendige Spannung verleihen, um ihrer Funktion wieder nachzukommen [8]

\section{Implantate}

Abb. 8-9 zeigen das Beispiel einer Ulnakopfprothese. Die Prothese besteht aus zwei Komponenten [7]:

Der Prothesenkopf hat eine sphärische Ballform, der distal zur Abstützung des TFCC und proximal zur Verbindung mit dem Prothesenschaft abgeflacht ist. Das Material ist Keramik (Zirkonoxid) und 


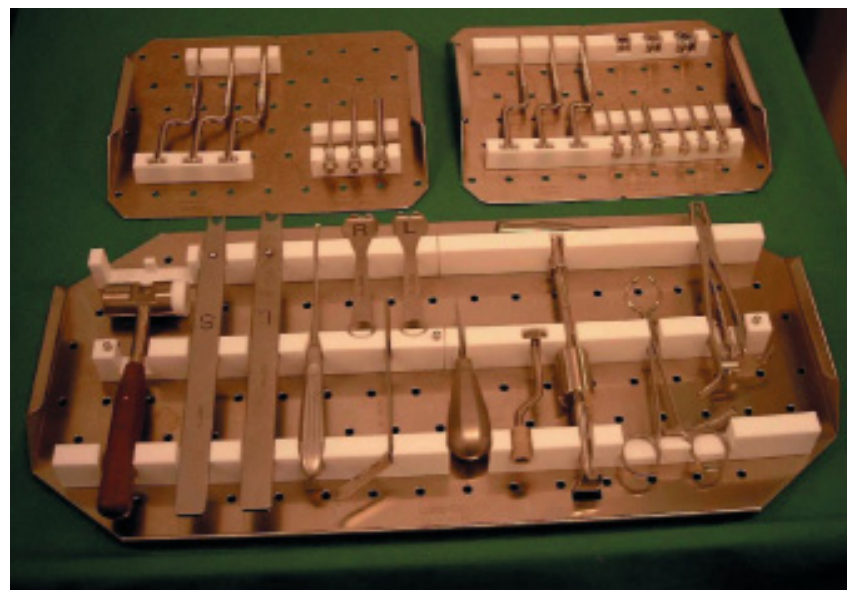

Abb. 7 Instrumentarium zur Implantation der Ulnakopfprothese (Fa. Martin, Tuttlingen). Die oberste Reihe zeigt Formraspeln, Probeschäfte und Probeköpfe.

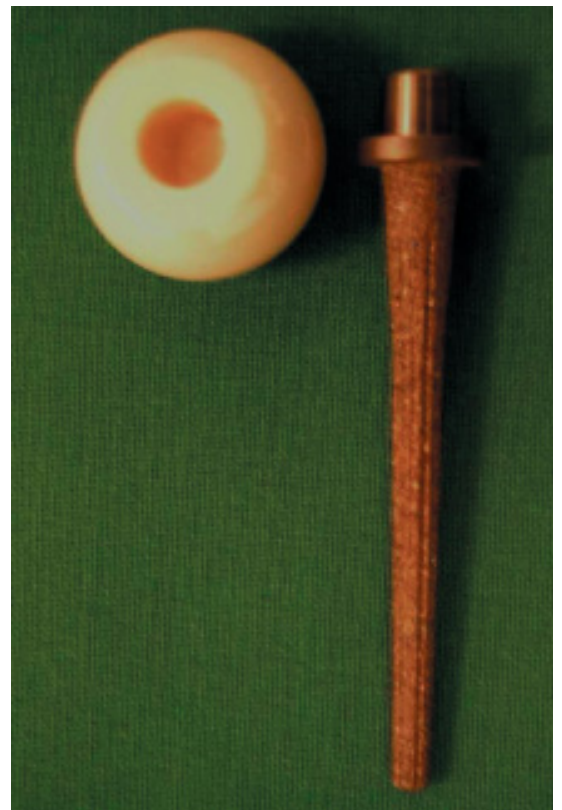

Abb. 8 Komponenten der Ulnakopfprothese (Fa. Martin, Tuttlingen).

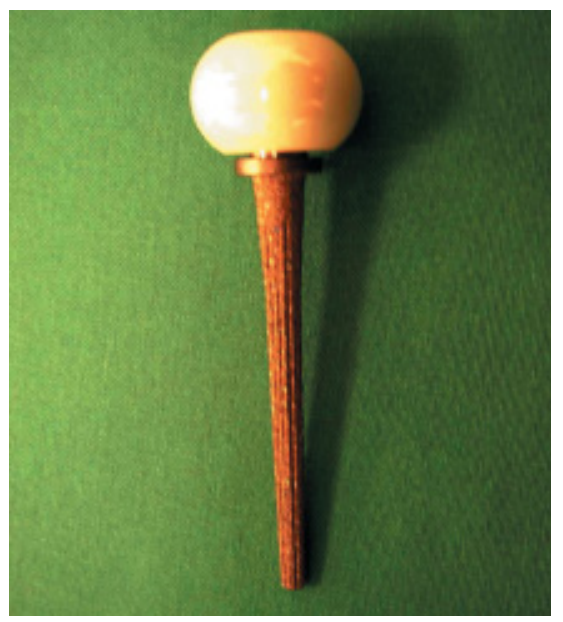

Abb.9 Montierte Ulnakopfprothese (Fa. Martin, Tuttlingen). es gibt drei Kopfgrößen. Der Prothesenschaft besteht aus Reintitan mit einer aufgerauhten, gekörnten Reintitanbeschichtung, welche eine knöcherne Integration im Ulnaschaft erlaubt. Auch für den Prothesenschaft liegen drei Größen vor. Die drei Schafttypen unterscheiden sich durch die Länge des Kragens, der sich zwischen Prothesenschaft und Prothesenkopf befindet. Die verschiedenen Kragenlängen erlauben eine Längenwiederherstellung der Ulna bei Substanzverlusten nach vorausgegangenen Resektionen.

\section{Voraussetzungen}

Eine Versorgung mit Ulnakopfprothese erfordert eine ausreichend tragfähige Ulna. Fehlende Länge kann in gewissen Grenzen durch Spezialanfertigung überlanger Prothesen ausgeglichen werden. Die Führung der Prothese erfolgt durch die umliegenden Weichteile, die intakt sein müssen (siehe Implantationstechnik). Um die axiale Stabilität zu gewährleisten müssen Humeroradialgelenk, proximales Radioulnargelenk und Membragegeben ist (z.B. fehlverheilte Radiuskopffrakturen, Essex-Lopresti-Läsionen, Situationen mit Verlust des Radiuskopfes) sind zunächst die Stabilitätsprobleme des Radius zu lösen, z. B. durch Implantation einer Radiuskopfprothese.

\section{Implantationstechnik [7]}

Die Implantation erfolgt über eine Längsinzision in Höhe des fünften Strecksehnenfaches, das eröffnet wird. Nach Retraktion der Extensor-digiti-minimiSehne werden der Boden des 5. Strecksehnenfaches und die Kapsel des DRUG bogenförmig inzidiert. Die ulnare Begrenzung bildet das sechste Strecksehna interossea intakt sein. Wo dies nicht nenfach, mit der Extensor carpi ulnaris Sehne, dass nicht eröffnet wird. Proximal endet die Inzision über der dorsalen Ulna, distal über dem Triquetrum. Retinakulum und Kapsel werden in einer Schicht vom Ulnakopf und der dorsalen Begrenzung des TFCC abpräpariert, wobei distal das ulnokarpale Handgelenk mit eröffnet wird. Hierdurch wird ein ulnar gestielter Lappen erzielt. Nach Festlegung der Resektionshöhe erfolgt die Osteotomie. Die Entfernung des Ulnakopfes erfolgt subperiostal ohne Verletzung des sechsten Strecksehnenfaches und distal ohne Beschädigung des TFCC. Der TFCC wird inspiziert und vorhandene Einrisse werden genäht. Der Ulnaschaft wird mit entsprechenden Formraspeln aufpräpariert und eine Probeprothese der vorbestimmten Größe eingebracht. Anhand der intraoperativen Bildwandlerkontrolle wird der Sitz der Prothese und das Niveau des Prothesenkopfes in Relation zur Radiusgelenkfläche in Neutralstellung, Pronation und Supination überprüft. Die Prothese sollte eine Ulna-minus-Situation von 1-2 mm in neutraler Rotationsstellung des Unterarmes herstellen. Korrekturen des Längenniveaus müssen $\mathrm{zu}$ diesem Zeitpunkt durch Nachresektion der distalen Ulna erfolgen. Nach Entfernung der Probeprothese werden zwei Bohrkanäle durch den dorsalen Rand der Incisura ulnaris des Radius gebohrt. Der Weichteillappen wird nach der Prothesenimplantation am dorsalen Rand des TFCC und transossär durch die vorgelegten Bohrkanäle im Radius refixiert. Die weitere Adaptation erfolgt am ulnaren Rand des Retinakulum extensorum des vierten Strecksehnenfaches. Die Extensor-digiti-minimi-Sehne wird subkutan belassen.

\section{Nachbehandlung}

Die postoperative Ruhigstellung erfolgt für zwei Wochen in einer Oberarmgipsschiene. Anschließend wird die Unterarmdrehbewegung für weitere vier Wochen durch eine ulnar umgreifende Thermoplastschiene limitiert.

\section{Ergebnisse}

Der Einsatz der Ulnakopfprothese löst

- sofern die oben genannten Voraussetzungen gegeben sind

च in den meisten Fällen die schmerzhafte Instabilitätsproblematik des distalen Ulnaendes nach fehlgeschlagenen Resektionsarthroplastiken oder schmerzhaften Arthrosen des DRUG [7]. 


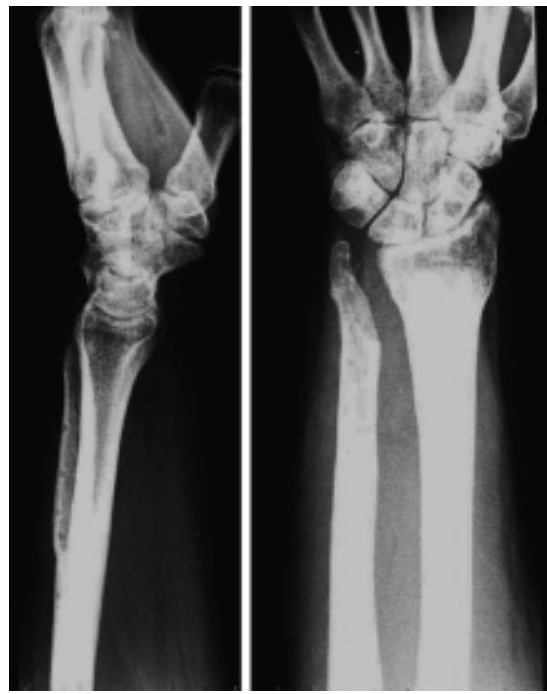

Abb.10 Handgelenk nach Ulnakopfhemiresektion.

Der Bewegungsumfang nimmt in der Regel deutlich zu. Ausgewählte Patienten demonstrieren die hohe funktionelle Belastbarkeit dieser Versorgung (Abb.10-13).

\section{Kontraindikationen}

Bei Insuffizienz der Weichteile fehlt die Stabilisierung des versorgten DRUG. Diese Situation kann bei Patienten vorliegen, bei denen multiple Operationen durchgeführt wurden. Bei Patienten mit ausgeprägter Fehlstellung des distalen Radius nach fehlverheilten Radiusfrakturen kann eine kongruente Einstellung des Prothesenkopfes in die Incisura ulnaris nur durch eine gleichzeitige Radiuskorrekturosteotomie erzielt werden. Als Kontraindikation ist die ausgeprägte Osteoporose anzusehen, da hier eine Integration des Prothesenschaftes nicht zu erwarten ist.

\section{Handgelenksprothesen}

\section{Grundlagen}

Die Zirkumduktion der Hand wird durch das Zusammenspiel von Radiokarpalgelenk (zwischen Radius und erster Handwurzelknochenreihe) und Mediokarpalgelenk (zwischen erster und zweiter Handwurzelknochenreihe) vermittelt. Eine ungestörte Funktion ist von der Integrität vieler kleiner Gelenkflächen und Bandstrukturen abhängig.

\section{Indikation}

Ähnlich wie bei der Arthrodese des Handgelenkes ist das Ziel der Handge-

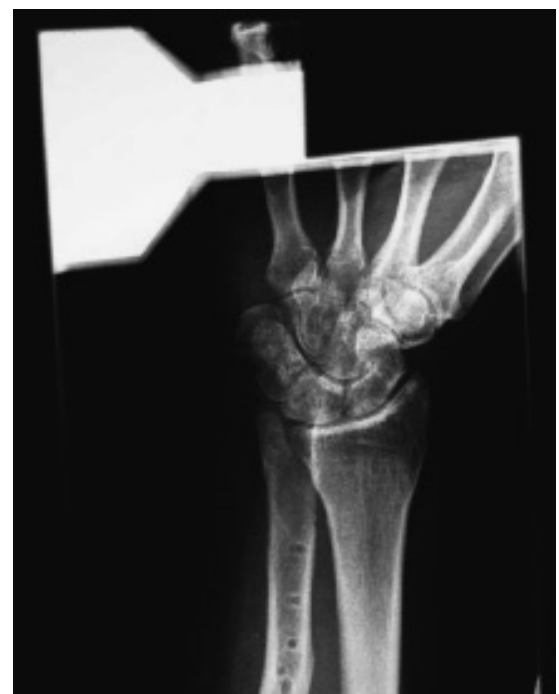

Abb.11 Unter Belastung zeigt sich die Instabilität der distalen Ulna mit Impingement.
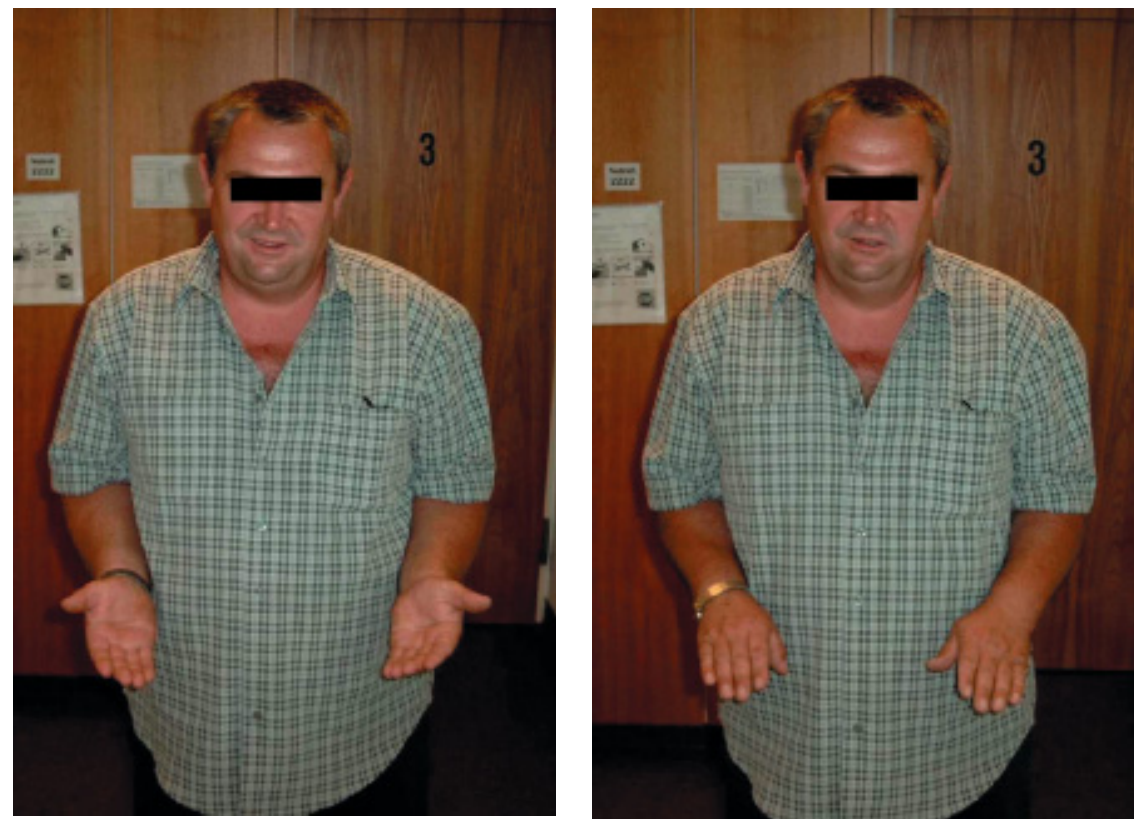

Abb.13a-b Gleicher Patient wie Abbildung 10-12. 2 Jahre nach Implantation einer Ulnakopfprothese auf der linken Seite übt der Patient seinen Beruf als Schmied vollschichtig aus. Die Unterarmdrehung ist frei.

lenksprothese die Beseitigung von Schmerzen, Instabilitäten und Deformitäten, wobei zusätzlich die Beweglichkeit erhalten bleiben soll. Eine Funktionsverbesserung kann dabei auch dadurch eintreten, dass die Weichteile durch die Normalisierung der Handwurzellänge besser vorgespannt werden [1]. Hauptindikation ist die Arthrose des Handgelenkes. Im Gegensatz zu Patienten mit Rheumatoidarthritis sind bei Patienten mit posttraumatischen Handgelenksveränderungen die proximalen Gelenke und die Muskel- spannung normal. Dies führt zu einer hohen mechanischen Belastung der Implantate und fördert das Implantatversagen. Da die Langzeitresultate der bisher zur Verfügung stehenden Implantate noch ungünstig sind, wird eine prothetische Versorgung in erster Linie bei zusätzlichen Bewegungseinschränkungen im Schulter-, Ellenbogen- und Fingergelenkbereich empfohlen oder bei beidhändigen Läsionen, um eine bilaterale Versteifung zu vermeiden [1]. 


\section{Vorraussetzungen}

Die Implatation setzt stabile knöcherne Verhältnisse und intakte Weichteilverhältnisse voraus. Um die Vorteile der Beweglichkeit ausnutzen zu können, müssen funktionsfähige Beuger und Strecker vorhanden sein.

\section{Implantationstechnik [1]}

Die Implantation erfolgt über einen dorsalen Zugang zum Handgelenk. Der distale Radius wird senkrecht zur Radiusachse reseziert. In einem weiteren Schnitt mit der oszillierenden Säge werden - je nach Prothesendesign in unterschiedlichem Ausmaß - Anteile der Handwurzel reseziert. Unter Umständen ist die zusätzliche Resektion des Ulnakopfes erforderlich. Die Implantation der distalen Prothesenkomponente erfolgt durch den Rest des Os capitatum im Verlauf des dritten Strahls (Drehachse des Handgelenkes) bis in Basis oder Schaft des dritten Mittelhandknochens.

\section{Ergebnisse}

Häufigste Probleme von Handgelenksprothesen sind die proximale und distale Lockerung der Prothesenschäfte, die zum Implantatversagen führen können $[1,10]$. Die Lockerungsraten betragen bis 53\% (nach [10]). Seltener treten Medianuskompressionen im Karpalkanal und Beugesehnenrupturen durch Reibung an der Prothese auf $[1,10]$.

Auch wenn einzelne Frühergebnisse Erfolg versprechend sind [2], ist die Indikation zur Handgelenksprothese sehr eng zu stellen. Eine vollständige Zerstörung des Handgelenkes und der Handwurzel ist selten. Bei solchen Extremverletzungen ist die Schädigung der Weichteile meistens so groß, dass hierdurch die Beweglichkeit limitiert wird, so dass eine Handgelenksprothese keine Verbesserung bringt. Häufiger sind arthrotische Spätfolgen umschriebener Läsionen am Handgelenk. Hierzu gehören auch die leicht zu übersehenden Bandverletzungen in der Handwurzel. Für solche Fälle stellen Teilarthrodesen eine erfolgversprechende Alternative dar. Bei diesen Verfahren wird - je nachdem, welches Teilgelenk betroffen ist - eine Fusion der angrenzenden Handwurzelknochen untereinander oder mit dem distalen Ra- dius durchgeführt. Da sich die Lastverteilung im Handgelenk dadurch nachhaltig ändert, handelt es sich funktionell um einen regelrechten biologischen Gelenkersatz, der eine vollständige Versteifung des Handgelenkes - bei einer gewissen Bewegungseinschränkung - vermeidet. Einen ähnlichen Ansatz verfolgt die Entfernung der proximalen Handwurzelreihe (proximal row carpectomy).

\section{Schlussfolgerungen}

Wenn die Erhaltung oder Rekonstruktion eines Gelenkes an der Hand nicht möglich ist, besteht in ausgewählten Fällen die Möglichkeit einer prothetischen Versorgung. Diese Maßnahme wird selten im Rahmen der Erstversorgung durchgeführt. Mehr noch als in anderen Körperregionen ist die Funktion der Prothese von den umgebenden Weichteilgeweben abhängig. Dazu gehören intakte oder rekonstruierbare Kapsel-Bandstrukturen und gleitfähige Sehnen. Eine endoprothetische Versorgung der Fingerendgelenke wird wegen des geringen Zuwachses an Beweglichkeit selten durchgeführt. Beim Ersatz von Fingergrund- und Mittelgelenken wird ein Kompromiss zwischen Beweglichkeit und Belastbarkeit eingegangen. Bei hohen Ansprüchen an die Belastbarkeit ist weiterhin die Arthrodese zu bevorzugen. Ob der Einsatz neuer Materialien (z.B. Pyrocarbon) die abriebbedingten Probleme (Implantatlockerung, Umgebungsreaktion) lösen wird, kann zur Zeit noch nicht entschieden werden. Durchstrukturierte Nachbehandlungskonzepte tragen zum initialen Erfolg wesentlich bei.

Die Ulnakopfprothese stellt die Integrität des distalen Radioulnargelenkes wieder her. Ihr Einsatzbereich ist nicht nur die primäre Schädigung dieses Gelenkes, sondern auch der Ausgleich von Defekten und Instabilitäten nach fehlgeschlagenen resezierenden Operationsverfahren.

Aufgrund der bisher ungünstigen Langzeitergebnisse sollte der Einsatz von Handgelenksprothesen nur unter strenger Indikationsstellung erfolgen. Teilversteifungen des Handgelenkes erlauben eine differenzierte Behandlung vieler posttraumatischer Zustände am Radiokarpalgelenk und Mediokarpalgelenk ohne auf die Beweglichkeit im Handgelenk völlig verzichten zu müssen.
Sie bilden daher einen guten Kompromiss zwischen kompletter Arthrodese und Prothese und sind, wenn immer möglich, zu bevorzugen.

\section{Literatur}

${ }^{1}$ Berger RA, Beckenbaugh RD, Linscheid RL Arthroplasty in the hand and the wrist. In: Green DP, Hotchkiss RN, Pederson WC (eds.). Green's operative hand surgery. Churchill Livingston, New York 1999; 147-191

${ }^{2}$ Kretschmer F, Wannske M. Die BIAX-Handgelenksprothese als Alternative zur Arthrodese bei degenerativer und posttraumatischer Arthrose - Frühergebnisse bei $21 \mathrm{~Pa}$ tienten. Handchir Mikrochir Plast Chir 2003; 35: $31-42$

${ }^{3}$ Lang E, Schmidt A, Ishida A, Baumgärtler $\mathrm{H}$ Erfahrungen mit dem alloplastischen $\mathrm{Ge}$ lenkersatz am Mittelgelenk. Handchir Mikrochir Plast Chir 2000; 32: 44-50

${ }^{4}$ Linscheid RL. Implant arthroplasty of the hand: retrospective and prospective considerations. J Hand Surg Am 2000; 25(5): $796-816$

${ }^{5}$ Pellegrini VD, Burton RI. Osteoarthritis of the proximal interphalangeal joint of the hand: Arthroplasty or fusion? J Hand Surg Am 1990; 15: 194-209

${ }^{6}$ Sauerbier M, Cooney WP, Berger RA, Linscheid RL. Kompletter Oberflächenersatz des Fingermittelgelenkes - Langzeitresultate und chirurgische Technik. Handchir Mikrochir Plast Chir 2000; 32: 411-418

${ }^{7}$ Schoonhoven J van, Herbert TJ, Fernandez DL, Prommersberger KJ, Krimmer H. Ergebnisse der Ulnakopfprothese. Im Druck

${ }^{8}$ Schoonhoven J van, Krimmer H. Neue Konzepte der Endoprothetik des distalen Radioulnargelenkes. Handchir Mikrochir Plast Chir 1998; 30: 387-392

${ }^{9}$ Stütz N, Behrendt M, Müller-Zimmermann A, Krimmer H. Pyrocarbon-Prothesen zum Ersatz von Fingermittelgelenken - Erfahrungswerte nach einem Jahr. Im Druck

${ }^{10}$ Vögelin E, Nagy L. Fate of failed Meuli total wrist arthroplasty. J Hand Surg Br 2003; 28(1): $61-68$

\section{Dr. med. Thomas Pillukat}

Assistenzarzt

Dr. med. Nikolas Stütz

Assistenzarzt

Priv.-Doz. Dr. med.

Jörg van Schoonhoven

Oberarzt

Priv.-Doz. Dr. med.

Herrmann Krimmer

Leitender Oberarzt

Klinik für Handchirurgie

Salzburger Leite 1

D-97616 Bad Neustadt an der Saale 